\title{
Liquid Crystal Light Valves: a versatile platform for Nematicons
}

\author{
Gaetano Assanto, ${ }^{* 1}$ Armando Piccardi, ${ }^{1}$ Alessandro Alberucci, ${ }^{1}$ Stefania Residori ${ }^{2}$ and Umberto Bortolozzo ${ }^{2}$ \\ ${ }^{I}$ NooEL, Nonlinear Optics and Opto-Electronics Laboratory, University "Roma Tre", Rome, Italy \\ ${ }^{2}$ INLN, Université de Nice Sophia-Antipolis, CNRS, 1361 Route des Lucioles, 06560 Valbonne, France
}

Received October 6, 2009; accepted December 22, 2009; published December 31, 2009

\begin{abstract}
We illustrate the generation and control of optical spatial solitary waves (nematicons) in liquid crystal light valves with nematic liquid crystals and a photoconductive layer. The latter allows to alloptically varying the birefringent walk-off and the extraordinary-wave refractive index, hence, the trajectory of a nematicon propagating in the thickness of the valve.
\end{abstract}

Spatial solitons in optics are one of the potential and most innovative all-optical routes for signal processing architectures. The control of their trajectories is relevant in reconfigurable interconnects [1-4]. In nematic liquid crystals, recent progress on spatial solitons ("Nematicons") and their control include electro-optic and opto-optical deviation and interactions, with overall changes in angle as large as 40 degrees [4-8].

Here we discuss excitation and tunable propagation of nematicons in photoconductive Light Valves with nematic Liquid Crystals (LCLV) [9-10], demonstrating a nonlinear dielectric setting where solitons can self-trap and propagate while interacting with externally defined refractive index perturbations. We show that nematicons in LCLV can be effectively launched and deviated according to the applied voltage as well as to external light illuminating the valve, thereby entailing complete control of the self-configured guided-wave circuitry.

The LCLV consisted of a glass slide and a photoconductor slab (BSO), separated by $\mathrm{L}=50 \mu \mathrm{m}$ and both coated with a layer of Indium Tin Oxide (ITO) in order to apply an external voltage $\mathrm{V}_{\text {bias }}$ (Fig. 1(a)) [9-10]. All surfaces were coated and mechanically rubbed with micro-grooves parallel to $y$ at the input facet and at $\pi / 4$ with respect to $z$ on the valve plates (Fig. 1(b)). The nematic liquid crystal (LC) we used was the mixture E48.

Nematicons were excited along $\boldsymbol{z}$ by a red beam (He$\mathrm{Ne}$ ) of waist of $\approx 5 \mu \mathrm{m}$. When extraordinarily polarized, the input beam propagated with a significant birefringent walk-off with respect to the input wave-vector parallel to $\boldsymbol{z}[5,11-12]$. For powers $\geq 1.5 \mathrm{~mW}$ the beam gave rise to a self-confined non spreading spatial soliton, which could propagate for over $>15$ Rayleigh lengths and at a walkoff $\delta_{0} \approx 7^{\circ}$, as sketched in Fig. 1(b) [12]. Photographs of

*E-mail: assanto@uniroma3.it the beam propagation for ordinary, mixed, extraordinary (e-) polarizations are shown in Fig. 2(a thru c).

Around an input power of $2 \mathrm{~mW}$, a nematicon was generated, as visible in Fig. $2(\mathrm{~d})$, propagating at $7^{\circ}$ with respect to $z$ and the ordinarily-polarized component (Fig. 2 (a)). Such angle could be reduced by varying the applied voltage and therefore the orientation of the optic axis, [5] making the walk-off (and the apparent walk-off in the observation plane $\boldsymbol{y} \boldsymbol{z}$ ) approach $0^{\circ}$ for $\mathrm{V} \approx 10 \mathrm{~V}$, as displayed in the photos and graph in Fig. 3.

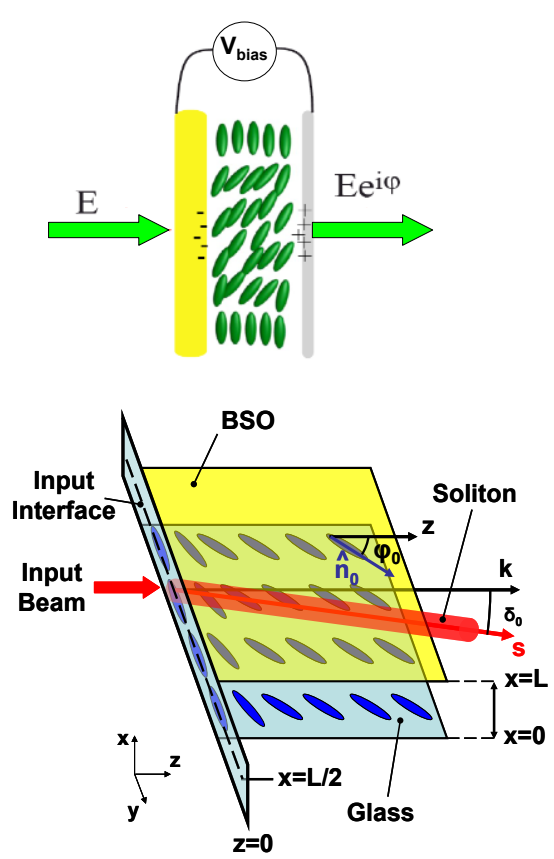

Fig.1. (a) Sketch of a liquid crystal light valve in a standard configuration for light transmission/modulation. The applied voltage $V_{0}$ varies the optical path, hence the phase of the transmitted light. (b) Launch configuration for nematicon generation and walk-off control. The director $\boldsymbol{n}$ is initially planar, laying in $\mathbf{y z}$ at $\varphi_{0}=\pi / 4$ with respect to $\mathbf{z}$. $\delta_{0}$ is the initial walk-off. 


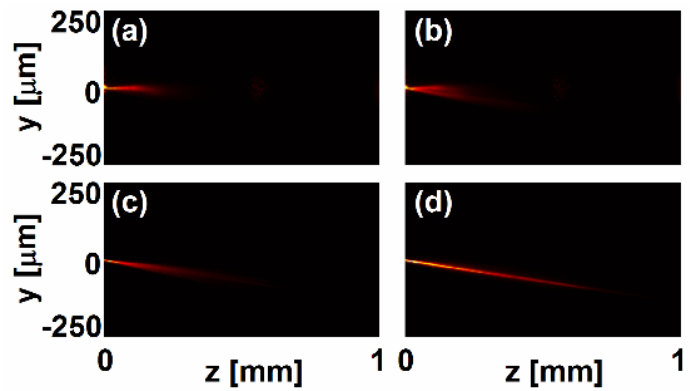

Fig. 2. Experiments on nematicons in an LCLV. (a) Ordinary wave beam excitation with input power $\mathrm{P}=2 \mathrm{~mW}$ : the beam diffracts as it propagates along its wavevector $\boldsymbol{k} / / \mathbf{z}$. (b) Mixed (ordinary and extraordinary) polarization beam of power $\mathrm{P}=2.5 \mathrm{~mW}$ launched in the LCLV: the ordinary component diffracts along $\boldsymbol{k}$ whereas the extraordinary one propagates along the Poynting vector $s$ at the walkoff angle. (c) An extraordinary wave beam with $\mathrm{P}<1 \mathrm{~mW}$ self-focuses as its Poynting vector walks off the $\boldsymbol{k}$ direction. (d) Extraordinary wave beam generating a nematicon for an input power $\mathrm{P}=2 \mathrm{~mW}$. The nematicon walks off at about 7 degrees with respect to the $\mathbf{z}$ axis.

(a)

(b)

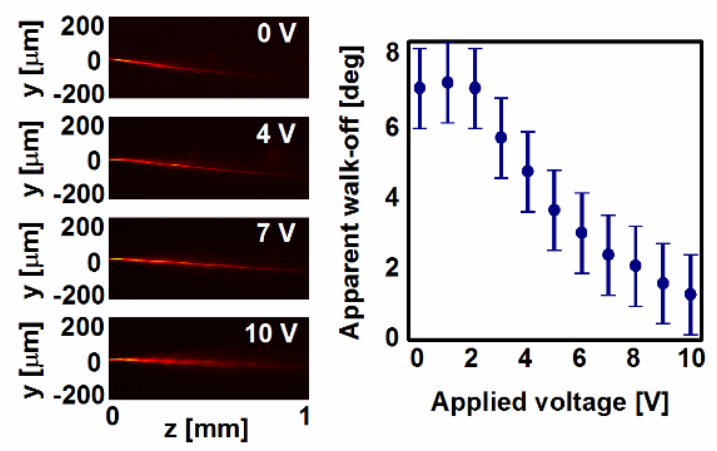

Fig. 3. Experimental results on controlling nematicon walk-off versus voltage in a biased LCLV. (a) Solitary light beam propagation for various applied biases, (b) apparent walk-off in the plane yz versus applied voltage $\mathrm{V}_{\text {bias }}$.

Using light at $532 \mathrm{~nm}$, i.e. at the wavelength at which the BSO is most sensitive, we could externally illuminate the valve and increase the voltage drop across the liquid crystal layer, due to the light-induced decrease in the photoconductor resistivity. Fig. 4 shows the changes in soliton walk-off via such light control. The measured change in direction of (energy) propagation could be fitted using a plane-wave model with an intensitydependent expression for the bias

$$
\mathrm{V}_{\mathrm{NLC}}=\Gamma \mathrm{V}_{\text {bias }}+\alpha \mathrm{I}
$$

with $\mathrm{V}_{\mathrm{NLC}}$ an effective voltage across the LC layer, $\mathrm{V}_{\text {bias }}$ the LCLV bias, I the external illumination intensity, $\Gamma$ and $\alpha$ suitable phenomenological constants [12].

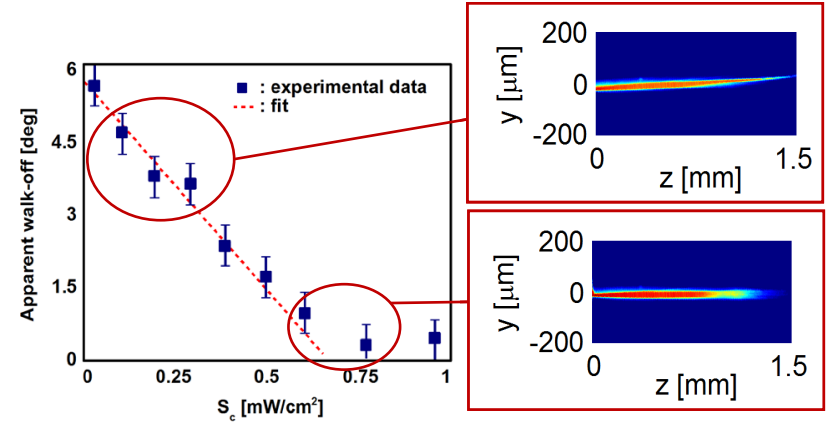

Fig. 4. Experimental results on controlling nematicon path by external illumination of intensity $\mathrm{S}_{\mathrm{c}}$ at $532 \mathrm{~nm}$ in a biased LCLV with $\mathrm{V}_{\text {bias }}=3.5 \mathrm{~V}$. The $2 \mathrm{~mW} e$-soliton changes direction of propagation (see insets) as the walk-off is modified by the control light with a resulting change in $\mathrm{V}_{\mathrm{NLC}}$.

Due to the RC constant introduced by the photoconductive layer and liquid crystals between the electrodes, the overall response of the light valve under bias was frequency limited to a few hundred Hertz, as apparent in Fig. 5 which shows the apparent walk-off under bias and illumination versus the frequency of the applied voltage. Clearly, no change in walk-off is obtained above $500 \mathrm{~Hz}$.

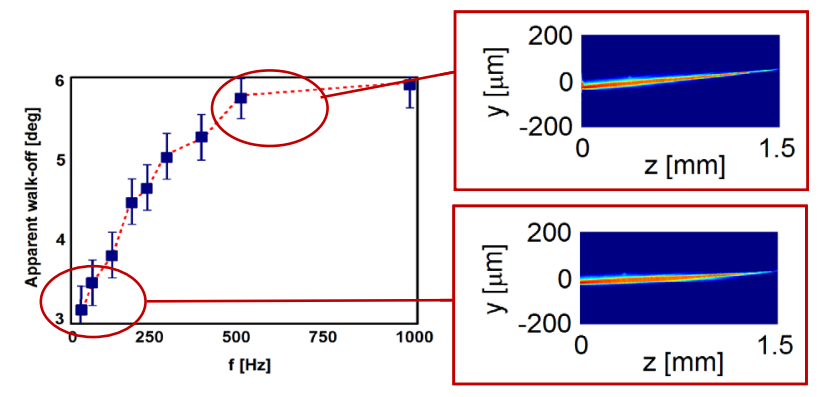

Fig. 5. Frequency dependence on controlling nematicon walk-off. The valve (with external illumination and a modulated bias of $3.5 \mathrm{~V}$ ) acts as a low pass filter; hence, it is no longer able to modify the $2 \mathrm{~mW}$ soliton trajectory above a cutoff $(1 / \mathrm{e})$ frequency of about $220 \mathrm{~Hz}$.

Finally, rather than a uniform illumination of the BSO layer, we employed finite size external beams at $532 \mathrm{~nm}$ to induce lens-like index/walk-off perturbation along the path of nematicons in the valve, as sketched in Fig. 6.

Solitons propagating in the vicinity of a light-induced defect could sense an increase in refractive index and a local variation of walk-off, bending their trajectories along paths depending on their relative distance from the perturbation, its size and shape. Using Eq. (1) above, the propagation of nematicons nearby externally-induced 


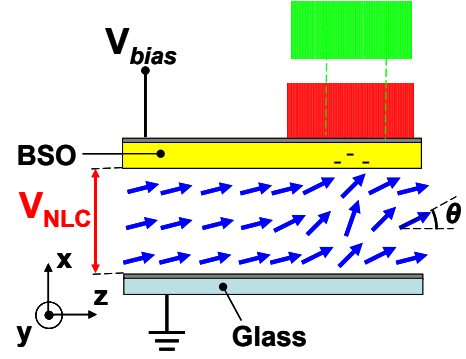

Fig. 6. Sketch of an LCLV for nematicon excitation and control. The soliton is excited by an extraordinarily-polarized light beam injected from the side of the valve. $V_{\text {bias }}$ is the external voltage bias, $V_{\mathrm{NLC}}$ the voltage drop across the nematic liquid crystal thickness, BSO indicates the photoconductive layer, the profile in green the external illumination, the profile in red the corresponding voltage change. The arrows represent the distribution of the LC molecular director, at the angle $\theta$ with respect to $z$.

"defects" along a local longitudinal coordinate $z$ could be modeled by two coupled partial differential equations for the director distortion $\theta_{V C}$ induced (in $\boldsymbol{x} \boldsymbol{z}$ ) by the bias and the control beam, and the evolution of the beam amplitude $A$ :

$\nabla^{2} \theta_{V C}+\gamma \sin \left(2 \theta_{V C}\right)\left(\frac{\Gamma V_{\text {bias }}+\alpha I}{L}\right)^{2}=0$

$2 \operatorname{in}_{e} k_{0}\left(\frac{\partial A}{\partial z_{l}}+\tan \delta \frac{\partial A}{\partial y_{l}}\right)+D_{x} \frac{\partial^{2} A}{\partial x_{l}^{2}}+D_{y} \frac{\partial^{2} A}{\partial y_{l}^{2}}+k_{0}^{2} \Delta n_{e}^{2} A=0$

with $\gamma=\Delta \varepsilon /(4 K), K$ the Frank elastic constant (taken equal for splay or bend or twist of the LC molecules) for the liquid crystal, $D_{x}$ and $D_{y}$ the diffraction coefficients, $\Gamma$ and $\alpha$ defined by Eq. (1), $k_{0}$ the propagation constant in vacuum, $\Delta \varepsilon$ the low-frequency anisotropy, $\Delta n_{e}^{2}$ the linear and nonlinear variation on the extraordinary refractive index $n_{e}, \delta$ the local walk-off and $L$ the distance between the ITO electrodes. The reference system $x_{l} y_{l} z_{l}$ is a moving frame rotating with the beam such that $z_{l}$ and $y_{l}$ are always parallel to the local wavevector and displacement electric vector, respectively. An example of the measured and simulated nematicon bending in the light valve near a light-induced lens-like perturbation is visible in Fig. 7 (top). The agreement between model and experiment is excellent. Fig. 7 (bottom panel) displays the three-dimensional trajectory of a nematicon under the influence of the transverse electric potential (bias) and the defect (vertical arrow). Owing to walk-off and refractive index perturbation, the soliton propagates oscillating up and down in the valve while bending its trajectory; hence, it moves along a complex 3D path deformable by both external bias and illumination.

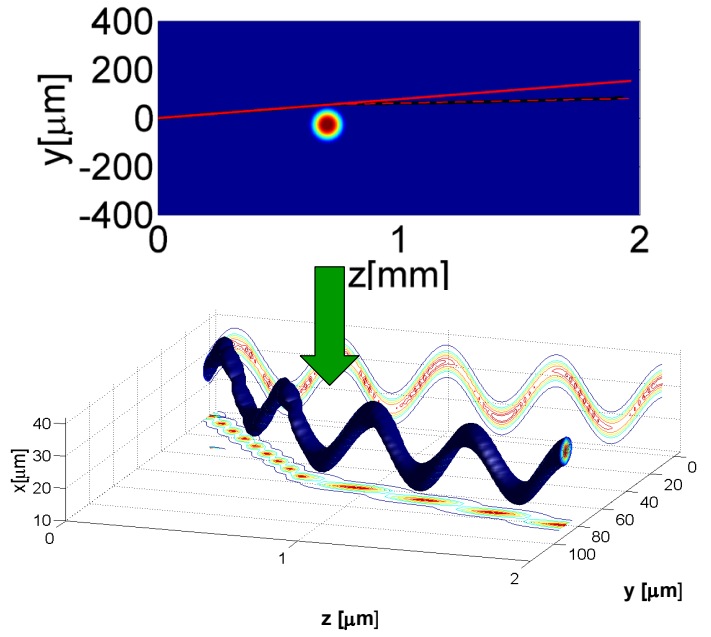

Fig. 7. Experimental results on controlling nematicon trajectory with an external control beam in a biased $50 \mu \mathrm{m}$ thick LCLV. Top: measured (black line) and calculated (dashed) nematicon trajectory in the proximity of a round all-optically induced perturbation. The red solid line refers to the unperturbed case. Bottom: computed 3D graph of the soliton path around the light-driven defect (arrow), in the presence of walk-off and a voltage-induced potential in $\mathbf{x z}$.

In conclusion, photoconductive liquid crystal light valves appear as an ideal environment for nematicon generation, propagation and trajectory control upon the application of external voltage and light. A number of alloptical operations can therefore be envisaged and will be demonstrated.

We thank the Galileo program 2008-2009 and the Italian-French University for financial support.

\section{References}

[1] A. W. Snyder, D. J. Mitchell and F. Ladouceur, Opt. Lett. 10, 21 (1991)

[2] N. N. Akhmediev, Opt. Quantum Electron. 30, 535-540 (1998)

[3] G. I. Stegeman and M. Segev, Science 286, 1518 (1999)

[4] C. Conti and G. Assanto, in Encyclopedia of Modern Optics, Vol. 5, pp. 43-55, edited by R. D. Guenther, D. G. Steel and L. Bayvel, Elsevier (Oxford, 2004)

[5] M. Peccianti, C. Conti, G. Assanto, A. De Luca and C. Umeton, Nature 432, 733 (2004).

[6] M. Peccianti, G. Assanto, A. Dyadyusha and M. Kaczmarek, Nat. Phys. 2, 737 (2006).

[7] M. Peccianti, G. Assanto, A. Dyadyusha and M. Kaczmarek, Phys. Rev. Lett. 98, 113902 (2007)

[8] M. Peccianti, A. Dyadyusha, M. Kaczmarek, and G. Assanto, Phys. Rev. Lett. 101, 153902 (2008)

[9] U. Bortolozzo, S. Residori and J. P. Huignard, J. Phys. D: Appl. Phys. 41, 224007 (2008)

[10] U. Bortolozzo, S. Residori, A. Petrosyan, and J. P. Huignard, Opt. Commun. 263, 317 (2006).

[11] M. Peccianti and G. Assanto, Opt. Lett. 30, 2290 (2005).

[12] A. Piccardi, U. Bortolozzo, S. Residori and G. Assanto, Opt. Lett. 34, 737 (2009).

[13] M. Peccianti, G. Assanto, A. De Luca, C. Umeton, I. C. Khoo, Appl. Phys. Lett. 77, 7 (2000).

[14] G. Assanto and M. Peccianti, Mol. Cryst. Liq. Cryst. 488, 163 (2008) 